
1. Capilla
2. Salas
3. Colegio
4. Casa congregación
5. Patio techado
6. Edificio preexistente

1. Asamblea

2. Presbiterio

3. Velatorio

4. Sacristía

5. Acceso

6. Confesionario

7. Sala de reunión

8. Coro

9. Asamblea

Decimos esto porque esta capilla difícilmente hubiéramos podido concebirla sin la tradición abierta en nuestro país dada por ejemplos como la capilla en el fundo Los Pajaritos del Instituto de Arquitectura de la Pontificia Universidad Católica de Valparaíso de 1953 (Cruz, 1954) y la iglesia del Monasterio Benedictino de la Santísima Trinidad de 1962 en Las Condes, Santiago, de Martín Correa y Gabriel Guarda (Aravena, 2002-2003).

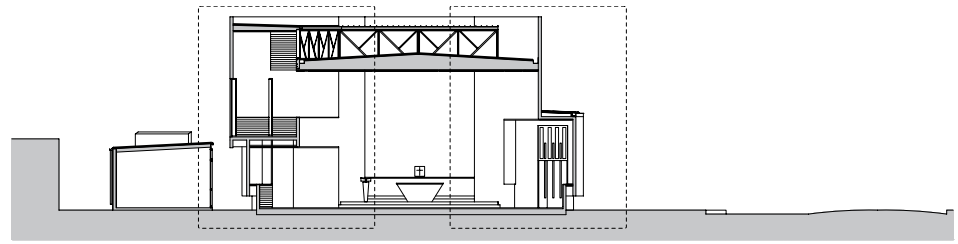

Corte AA

E. 1: 500 .

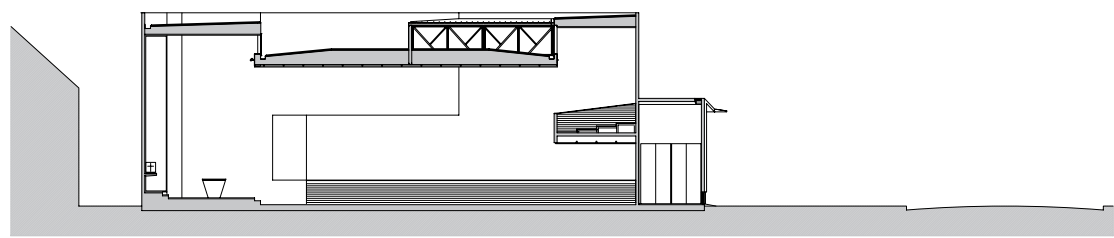

Corte BB

E. 1: 500 .
Avda. San Francisco



azamiento

E. 1: 2.500 .

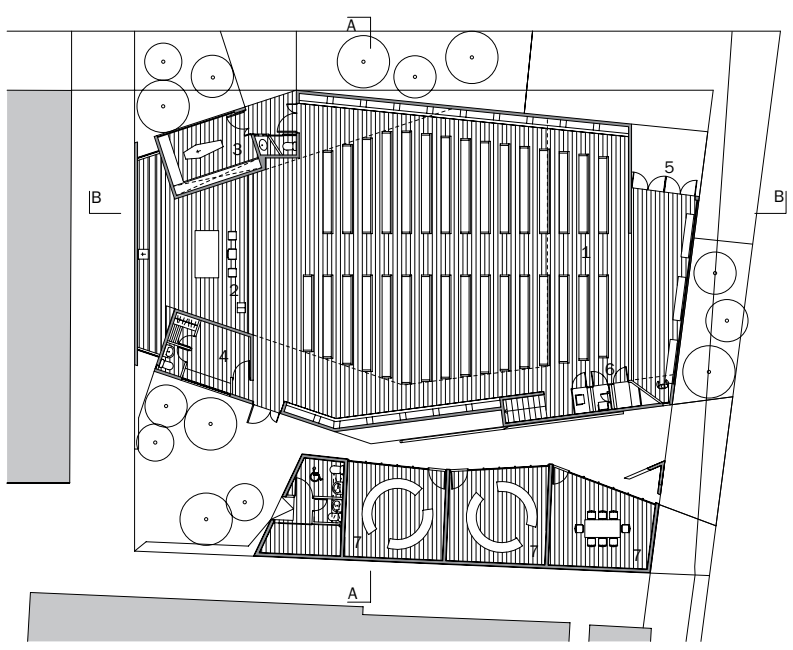

Planta primer piso

E. 1: 500 .

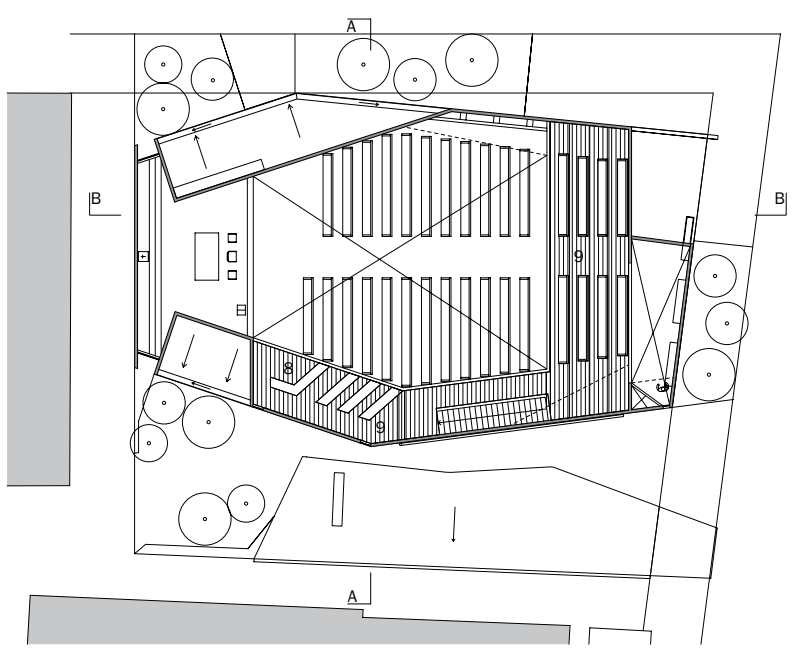

Planta segundo piso

E. 1: 500 . 

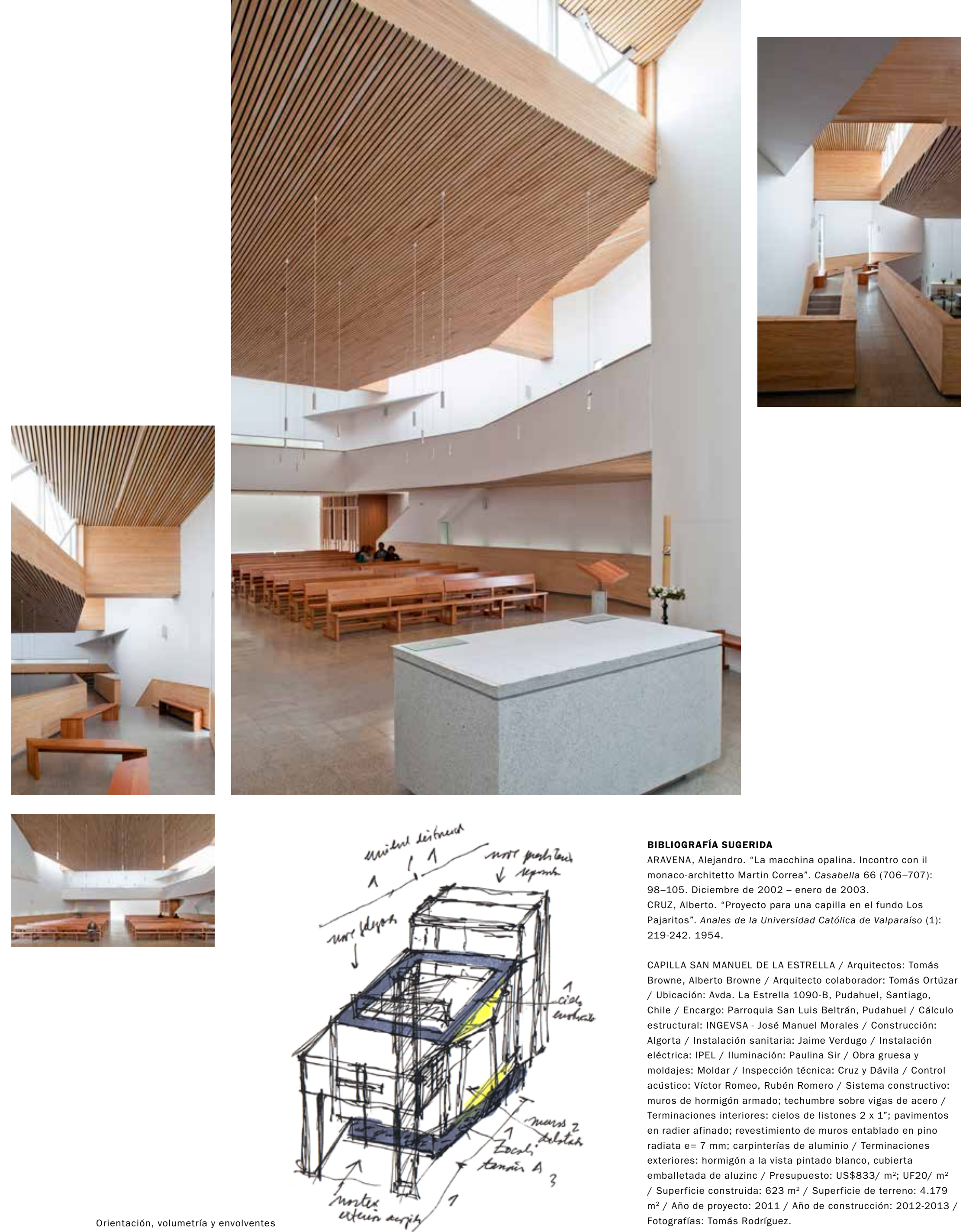

\section{BIBLIOGRAFÍA SUGERIDA}

ARAVENA, Alejandro "La macchina opalina. Incontro con il monaco-architetto Martin Correa". Casabella 66 (706-707): 98-105. Diciembre de 2002 - enero de 2003.

CRUZ, Alberto. "Proyecto para una capilla en el fundo Los Pajaritos". Anales de la Universidad Católica de Valparaíso (1) 219-242. 1954.

CAPILLA SAN MANUEL DE LA ESTRELLA / Arquitectos: Tomás Browne, Alberto Browne / Arquitecto colaborador: Tomás Ortúzar / Ubicación: Avda. La Estrella 1090-B, Pudahuel, Santiago, Chile / Encargo: Parroquia San Luis Beltrán, Pudahuel / Cálculo estructural: INGEVSA - José Manuel Morales / Construcción: Algorta / Instalación sanitaria: Jaime Verdugo / Instalación eléctrica: IPEL / Iluminación: Paulina Sir / Obra gruesa y moldajes: Moldar / Inspección técnica: Cruz y Dávila / Control acústico: Víctor Romeo, Rubén Romero / Sistema constructivo: muros de hormigón armado; techumbre sobre vigas de acero / Terminaciones interiores: cielos de listones $2 \times 1$ "; pavimentos en radier afinado; revestimiento de muros entablado en pino radiata $\mathrm{e}=7 \mathrm{~mm}$; carpinterías de aluminio / Terminaciones exteriores: hormigón a la vista pintado blanco, cubierta emballetada de aluzinc / Presupuesto: US $\$ 833 / \mathrm{m}^{2}$; UF20/ $\mathrm{m}^{2}$ / Superficie construida: $623 \mathrm{~m}^{2}$ / Superficie de terreno: 4.179 m² / Año de proyecto: 2011 / Año de construcción: 2012-2013 / Fotografías: Tomás Rodríguez. 